\title{
SIMPLIFIED CRACK INITIATION ANALYSIS OF PIEZOELECTRIC CERAMICS UNDER CYCLIC LOADING
}

\author{
M. Mizuno ${ }^{1}$ and T. Shimomura ${ }^{2}$ \\ ${ }^{1}$ Department of Machine Intelligence and Systems Engineering, Akita Prefectural University, \\ Honjo, Akita 015-0055, Japan \\ ${ }^{2}$ Graduate Student of Akita Prefectural University, Honjo, Akita 015-0055, Japan
}

\begin{abstract}
Crack initiation of piezoelectric ceramics under cyclic loading conditions of mechanical and electric loads was analyzed by a double cantilever beam (DCB) model. The DCB model is a simplified method to analyze a macroscopic crack by using some assumptions, and the effects of important factors on the crack can be elucidated qualitatively. The author have so far proposed that damage generated in piezoelectric ceramics by mechanical and electric loads was represented by a damage variable on the basis of the continuum damage mechanics. It has been also proposed that the damage variable was introduced into a constitutive equation of piezoelectric ceramics by using the modified cubes model and an evolution equation of the damage variable was formulated. Then, the constitutive equation and the damage evolution equation were applied to the DCB model. In the analysis by using the DCB model, a criterion of the crack initiation was based on the continuum damage mechanics; i.e. crack initiation begins when the damage variable at the crack tip attains to the critical value. In the present study, cyclic loading conditions of mechanical and electric loads were taken into account in the DCB model. By using the DCB model, the number of cycles of cyclic loading until crack initiation (or crack initiation time), fracture strain at a crack tip, and distributions of stress, strain and damage variable in front of the crack tip until the crack initiation were calculated numerically, and the effects of applied electric field on them were discussed.
\end{abstract}

\section{INTRODUCTION}

Since piezoelectric ceramics are endowed with superior characteristics such as response at high speed, generation of high power in relative small volume and infinitesimal displacement by precise control, they are used in a variety of actuators. When the piezoelectric ceramics are used in high power actuators, they are subjected to both mechanical and electric loads cyclically during the operation. The mechanical and electric cyclic-loading cause damage development in the material gradually, and the damage development causes fracture at last.

The damage generated by mechanical and electric loading was represented by a damage variable on the basis of the continuum damage mechanics in order to describe a state of the damage developed in piezoelectric ceramics and to intend to apply the damage variable to an analysis of crack growth. Then, the author [1] have so far proposed a constitutive equation of piezoelectric ceramics into which the damage variable was incorporated by using the modified cubes model [2]. Moreover, an evolution equation of the damage variable has been formulated [1].

The constitutive equation and the damage evolution equation were applied to a double cantilever beam (DCB) model [3], and a simplified analysis of crack initiation of piezoelectric ceramics under constant mechanical and electric loads has been performed by the author [4]. The 
DCB model is a simplified method to analyze initiation and growth of a macroscopic crack in a plate by using some assumptions. By using the DCB model combined with the concept of the continuum damage mechanics [5], crack initiation time, crack growth rate, fracture strain at a crack tip and distributions of stress, strain and damage variable in front of the crack tip can be calculated by taking into account the effects of applied electric field.

In the present paper, crack initiation of piezoelectric ceramics is analyzed under various cyclic loading conditions of mechanical and electric loads by using the DCB model. Then, the number of cycles of cyclic loading until crack initiation and distributions of stress, strain and damage variable in front of the crack tip until the crack initiation are calculated, and the effects of applied electric field on the crack initiation under cyclic loading conditions are discussed.

\section{CONSTITUTIVE EQUATION AND DAMAGE EVOLUTION EQUATION}

In general, a constitutive equation of piezoelectric ceramics is given as follows:

$$
\begin{aligned}
\sigma_{i j} & =C_{i j k l} \varepsilon_{k l}-e_{m i j} E_{m}, \\
D_{i} & =e_{i k l} \varepsilon_{k l}+\kappa_{i m} E_{m},
\end{aligned}
$$

where $\sigma_{i j}, \varepsilon_{i j}, E_{i}$ and $D_{i}$ represent stress, strain, electric field and electric displacement, respectively, while $C_{i j k l}, e_{m i j}$ and $\kappa_{i m}$ are elastic modulus, piezoelectric modulus and permittivity, respectively.

Piezoelectric ceramics suffer damage generated by mechanical and electric loading, and the damage affects material constants in eqns (1) and (2). In order to introduce the effects of the damage into the constitutive equation (1) and (2), the damage state is represented by a damage variable $\omega$ based on the continuum damage mechanics [5, 6], and the material constants in eqns (1) and (2) are expressed by material functions of $\omega$ as follows [1]:

$$
C_{i j k l}=C_{i j k l}(\omega), e_{m i j}=e_{m i j}(\omega), \kappa_{i m}=\kappa_{i m}(\omega) .
$$

In order to formulate the material functions (3), the modified cubes model [2] is used. If the damage variable $\omega$ is interpreted as reduction of load-carrying net area caused by microscopic cavities [6], the material functions are given as follows [1]:

$$
\begin{gathered}
C_{3333}(\omega)=(1-\omega) C_{3333}^{0}, \\
e_{333}(\omega)=(1-\omega) e_{333}^{0}, \\
\kappa_{33}(\omega)=(1-\omega) \kappa_{33}^{0}+\frac{\omega \kappa_{33}^{\text {air }} \kappa_{33}^{0}}{\sqrt{\omega} \kappa_{33}^{0}+(1-\sqrt{\omega}) \kappa_{33}^{\text {air }}},
\end{gathered}
$$

where $C_{3333}^{0}, \quad e_{333}^{0}$ and $\kappa_{33}^{0}$ represent elastic, piezoelectric and dielectric constants of piezoelectric ceramics without any damage, respectively. $\kappa_{33}^{\text {air }}$ is permittivity of air in cavities.

An evolution equation of the damage variable is formulated by taking into account the effects of applied electric field on the damage development as well as the effects of mechanical loading as follows [1]:

$$
\frac{d \omega}{d t}=A\left[\frac{\alpha \sigma^{I}+(1-\alpha) \sigma^{E Q}+\beta|D|+\gamma\langle-D\rangle}{1-\omega}\right]^{k},
$$

where $\sigma^{I}$ and $\sigma^{E Q}$ represent the maximum principal stress and the equivalent stress, respectively. $\alpha$ is a material constant to represent the dependence of the damage development on a stress state. $A, k, \beta$ and $\gamma$ are material constants. In eqn (7), angular brackets \langle\rangle represent Macauley bracket meaning that $\langle x\rangle=0$ if $x<0,\langle x\rangle=x$ if $x \geq 0$. 


\section{METHOD OF CRACK INITIATION ANALYSIS BY DCB MODEL}

In the DCB model [3], a plate with a crack is divided into three zones as shown in Figure 1; i.e. an elastic zone $\Omega_{E}$, a fractured zone $\Omega_{F}$ and a damaging zone $\Omega_{D}$. The elastic zone $\Omega_{E}$ is treated as a cantilever beam in which elastic shear deformation is dominant, while a crack is included in the fractured zone $\Omega_{F}$ where any deformation is not considered. The constitutive equation (1)-(6) and the damage evolution equation (7) are applied to the damaging zone $\Omega_{D}$, in which a stress state is assumed to be uniaxial. In this analysis, poling direction coincides with $y$-axis in the coordinate system $o-x y$ in the figure, and applied electric field in the same direction as the poling direction is positive.

The relation between displacement and stress in the elastic zone $\Omega_{E}$ is given by [3]:

$$
\frac{\partial^{2} \varepsilon(x, t)}{\partial x^{2}}=\frac{\sigma(x, t)}{G_{0} h \lambda},
$$

where $G_{0}\left(=C_{1133}^{0}\right.$ in the notation of eqns (1) and (2)), $h$ and $\lambda$ are the shear modulus, the width of the elastic zone $\Omega_{E}$ and that of the damaging zone $\Omega_{D}$, respectively.

Since a uniaxial stress state is assumed in the damaging zone $\Omega_{D}$, a constitutive equation and an evolution equation in $\Omega_{D}$ are expressed from eqns (1)-(3) and (7) as follows:

$$
\begin{gathered}
\sigma(x, t)=C(\omega(x, t)) \varepsilon(x, t)-e(\omega(x, t)) E(t), \\
D(x, t)=e(\omega(x, t)) \varepsilon(x, t)+\kappa(\omega(x, t)) E(t), \\
\frac{\partial \omega(x, t)}{\partial t}=A\left[\frac{\alpha\langle\sigma(x, t)\rangle+(1-\alpha)|\sigma(x, t)|+\beta|D(x, t)|+\langle-D(x, t)\rangle}{1-\omega(x, t)}\right]^{k},
\end{gathered}
$$

where material functions $C(\omega(x, t)), e(\omega(x, t))$ and $\kappa(\omega(x, t))$ are given by eqns (4)-(6).

An initial value of stress $\sigma(x, t)$, strain $\varepsilon(x, t)$ and damage variable $\omega(x, t)$ at time $t=0$ is given as follows:

$$
\sigma(x, t=0)=0, \varepsilon(x, t=0)=0, \omega(x, t=0)=0 .
$$

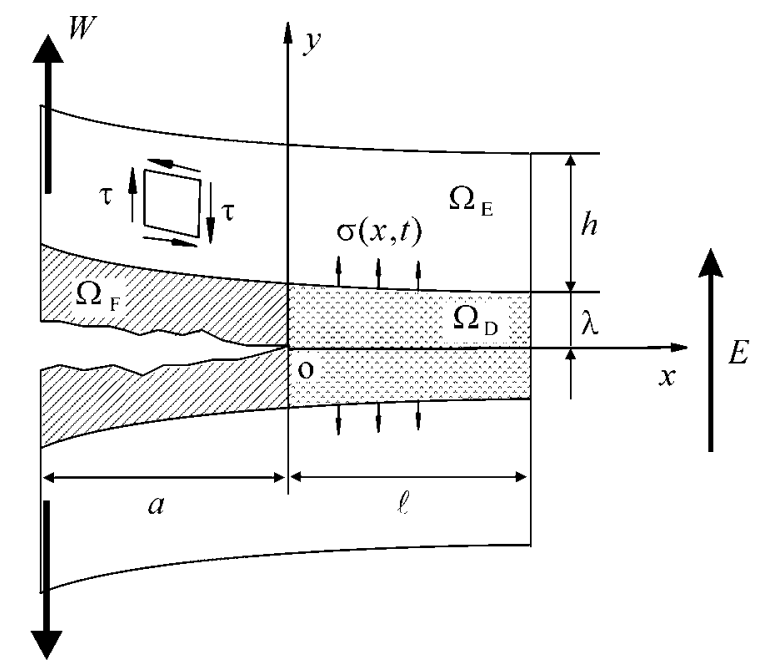

Figure 1 Double cantilever beam (DCB) model. 
When a point weight $W(t)$ is considered as shown in Figure 1, boundary conditions are given at a crack tip ( $x=0)$ and at the end of right hand side of the plate $(x=\ell)$ as follows [3]:

$$
\begin{gathered}
\frac{\partial \varepsilon}{\partial x}(x=0, t)=-\frac{W(t)}{G_{0} h \lambda}=-\frac{\tau_{0}(t)}{G_{0} \lambda}, \\
\frac{\partial \varepsilon}{\partial x}(x=\ell, t)=0, \quad \varepsilon(x=\ell, t)=\frac{e_{0}}{C_{0}} E(t),
\end{gathered}
$$

where $\tau_{0}(t)$ is shear stress at the crack tip, and $C_{0}=C_{3333}^{0}$ and $e_{0}=e_{333}^{0}$.

When a damage variable at the crack tip attains to 1 , the crack growth initiates. It is the crack initiation time $t_{i n i}$. Accordingly, the crack initiation time $t_{i n i}$ and rupture strain $\varepsilon_{R}$ at the crack tip are obtained by following conditions:

$$
\omega\left(x=0, t=t_{\text {ini }}\right)=1, \quad \varepsilon\left(x=0, t=t_{\text {ini }}\right)=\varepsilon_{R} .
$$

In the present paper, cyclic loading conditions of mechanical and electric loads are taken into account. In the present analysis, the shear stress at the crack tip $\tau_{0}(t)$ and applied electric field $E(t)$ are given by a sine function as follows:

$$
\tau_{0}(t)=\frac{\tau_{\max }}{2}\left[1+\sin \left(2 \pi f t-\frac{\pi}{2}\right)\right], E(t)=\frac{E_{\max }}{2}\left[1+\sin \left(2 \pi f t \pm \frac{\pi}{2}\right)\right],
$$

where $f$ is a frequency of applied loads cyclically. The electric field is applied so that it is synchronized with the mechanical load (In-phase), and it is applied with phase difference of $\pi$ from the phase of the mechanical load (Out-of-phase).

By solving equations (8)-(11) for the initial conditions (12) and the boundary conditions (13) and (14), distributions of stress $\sigma(x, t)$, strain $\varepsilon(x, t)$ and damage variable $\omega(x, t)$ in the direction of $x$-axis at arbitrary time $t$ in the damaging zone $\Omega_{D}$ are obtained. The equations (8)-(11) are solved repeatedly according to the passage of time $t$, then, the crack initiation time $t_{\text {ini }}$ and the rupture strain $\varepsilon_{R}$ at the crack tip are calculated when the conditions (15) are satisfied. In this study, equations (8)-(11) were solved by a numerical method.

\section{RESULTS OF ANALYSIS}

The analysis was performed for lead zirconate titanate (PZT) piezoelectric ceramics which was selected as typical piezoelectric ceramics. $\tau_{\max }$ in eqn (16) was changed systematically, and three kinds of applied electric field were given; i.e. $E_{\max }=0,2.0$ (In-phase), 2.0 (Out-of-phase) MV/m. Then, the effect of the applied electric field was elucidated.

Figure 2 shows the number of cycles of loading until crack initiation depending on applied electric field. The number of cycles in general increases when the maximum shear stress decreases. On the other hand, the number of cycles decreases when the electric field (In-phase) is applied, while the number of cycles does not change when the electric filed (Out-of-phase) is applied.

Figure 3 shows development of damage variable at the crack tip $\omega(x=0, t)$. A close-up of the development is inserted in the figure. The damage variable increases gradually cycle by cycle. The development is accelerated when the electric field (In-phase) is applied, while there is no effect of applied electric field (Out-of phase) on the development of the damage variable.

Figure 4 shows the distribution of damage variable in front of the crack tip until the crack initiation. A close-up of the distribution in the vicinity of the crack tip is inserted. The damage develops in the extremely narrow range near the crack tip. The value of the damage variable at the crack tip increases cycle by cycle, and the damage variable attains to 1 at last. 
Figure 5 shows distribution of stress in front of the crack tip until the crack initiation. A close-up of the distribution near the crack tip is inserted in the figure. There is a peak of the distribution near the crack tip. The value of the stress at the crack tip decreases to 0 cycle by cycle. The value of the peak decreases and the position of the peak shifts away far from the crack tip.

\section{ACKNOWLEDGMENT}

This work was supported by JSPS. KAKENHI (15560078).

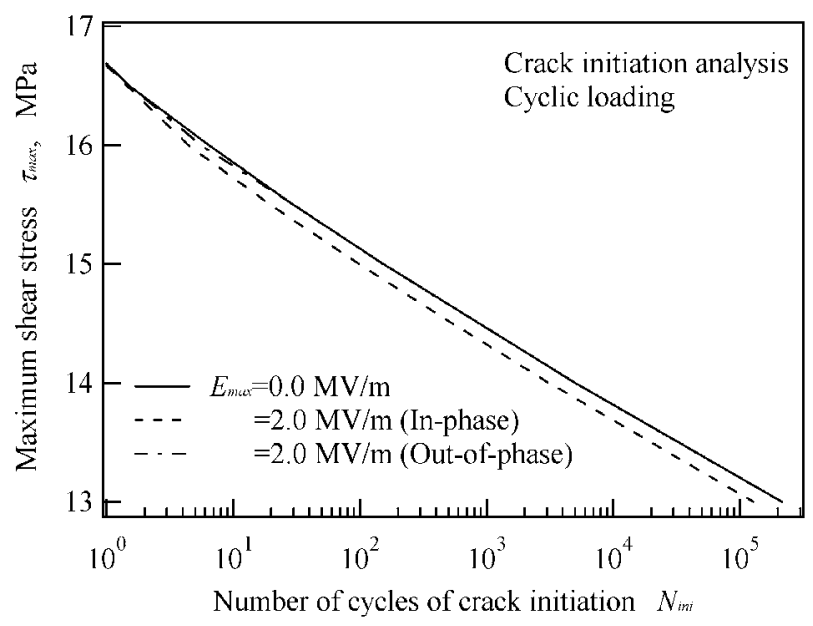

Figure 2 Number of cycles of loading until crack initiation depending on applied electric field.

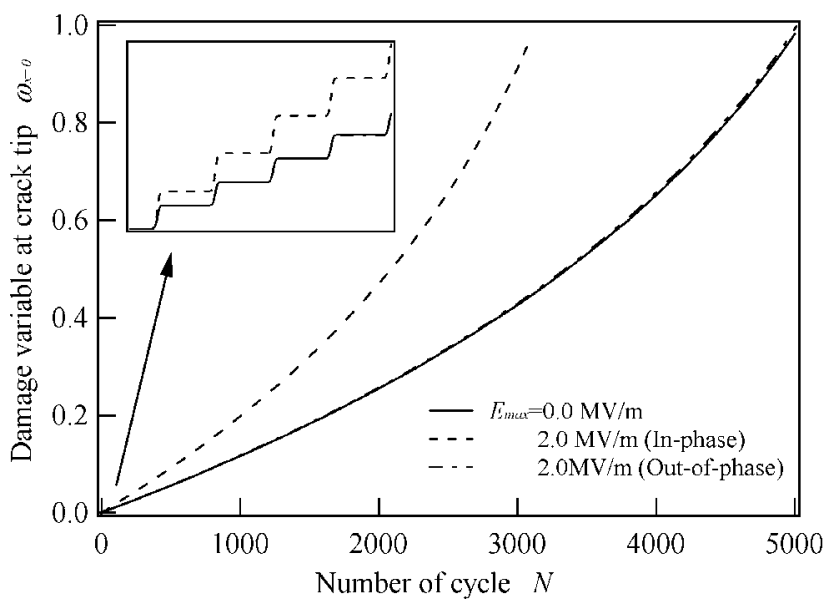

Figure 3 Development of damage variable at the crack tip cycle by cycle. 


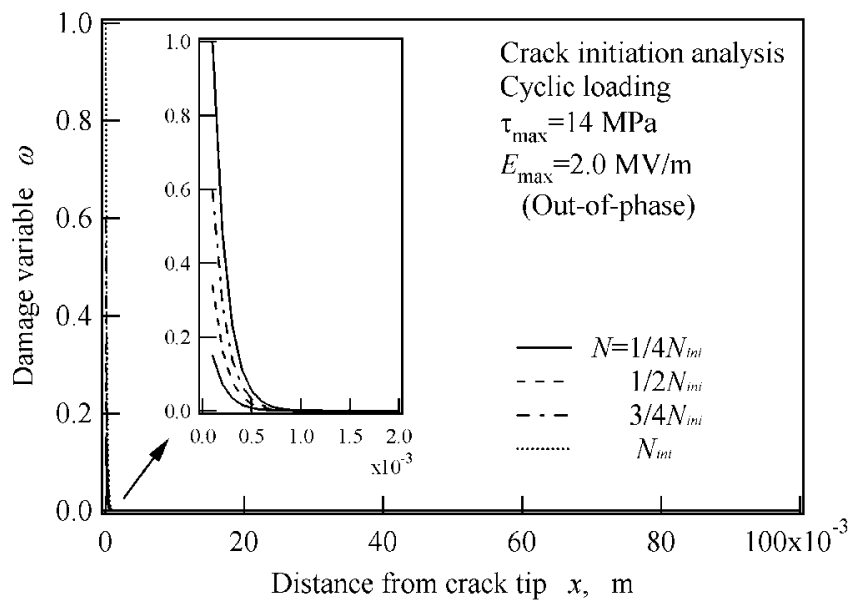

Figure 4 Distribution of damage variable in front of the crack tip until crack initiation.

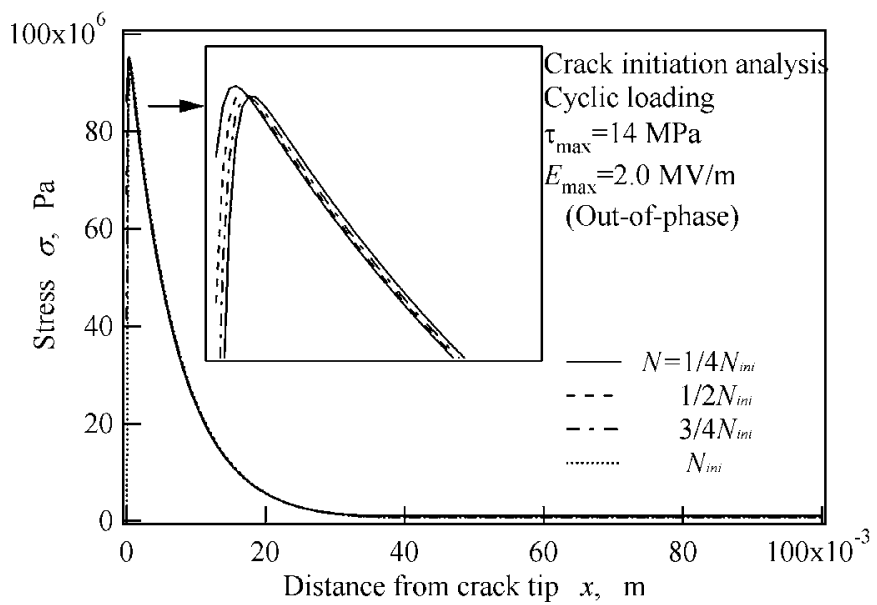

Figure 5 Distribution of stress in front of the crack tip until crack initiation.

\section{REFERENCES}

[1] Mizuno, M., Key Eng. Mater., vols. 233-236, pp. 89-94, 2003.

[2] Banno, H., Ceram. Bull., vol. 66, pp. 1332-1337, 1987.

[3] Murakami, S., Hayakawa, K. and Mizuno, M., Eur. J. Mech., A/Solids, vol. 12, pp. 249-263, 1993.

[4] Mizuno, M., Proc. 9th Int'l Conf. on the Mechanical Behaviour of Materials, (on CD-ROM), 2003.

[5] Lemaitre, J., A Course on Damage Mechanics, Springer-Verlag, 1992.

[6] Murakami, S., JSME Int'l. J., vol. 30, pp. 701-710, 1987. 